REVIEW

\title{
Autoregulation of hepatic glucose production
}

\author{
Mary Courtney Moore, Cynthia C Connolly and Alan D Cherrington \\ 702 Light Hall, Department of Molecular Physiology and Biophysics, Vanderbilt University School of Medicine, Nashville,
} Tennessee 37232-0615 USA

(Correspondence should be addressed to M C Moore)

\begin{abstract}
In vitro evidence indicates that the liver responds directly to changes in circulating glucose concentrations with reciprocal changes in glucose production and that this autoregulation plays a role in maintenance of normoglycemia. Under in vivo conditions it is difficult to separate the effects of glucose on neural regulation mediated by the central nervous system from its direct effect on the liver. Nevertheless, it is clear that nonhormonal mechanisms can cause significant changes in net hepatic glucose balance. In response to hyperglycemia, net hepatic glucose output can be decreased by as much as $60-90 \%$ by nonhormonal mechanisms. Under conditions in which hepatic glycogen stores are high (i.e. the overnight-fasted state), a decrease in the glycogenolytic rate and an increase in the rate of glucose cycling within the liver appear to be the explanation for the decrease in hepatic glucose output seen in response to hyperglycemia. During more prolonged fasting, when glycogen levels are reduced, a decrease in gluconeogenesis may occur as a part of the nonhormonal response to hyperglycemia.

A substantial role for hepatic autoregulation in the response to insulin-induced hypoglycemia is most clearly evident in severe hypoglycemia $(\leq 2.8 \mathrm{mmol} / \mathrm{l})$. The nonhormonal response to hypoglycemia apparently involves enhancement of both gluconeogenesis and glycogenolysis and is capable of supplying enough glucose to meet at least half of the requirement of the brain. The nonhormonal response can include neural signaling, as well as autoregulation. However, even in the absence of the ability to secrete counterregulatory hormones (glucocorticoids, catecholamines, and glucagon), dogs with denervated livers (to interrupt neural pathways between the liver and brain) were able to respond to hypoglycemia with increases in net hepatic glucose output. Thus, even though the endocrine system provides the primary response to changes in glycemia, autoregulation plays an important adjunctive role.
\end{abstract}

European Journal of Endocrinology 138 240-248

\section{Introduction}

The liver plays a unique role in the regulation of carbohydrate metabolism because it is able both to take up and release glucose, thus helping to dampen variations in blood glucose. For at least 60 years evidence has existed which suggests that the liver is able to respond directly to changes in blood glucose with reciprocal changes in hepatic glucose output (1), a phenomenon known as hepatic autoregulation.

In vitro data provide strong support for the existence of autoregulation. The perfused rat liver increases its rate of glucose output when the perfusate contains no glucose or a low glucose concentration and decreases glucose output when the perfusate glucose concentration is high (2-5). In vivo studies in dogs, miniature pigs, and humans support the concept of hepatic autoregulation.

\section{Autoregulation in response to hyperglycemia}

In studies conducted in the $36 \mathrm{~h}$ fasted conscious dog, Shulman et al. $(6,7)$ noted that hyperglycemia (plasma glucose $\sim 12 \mathrm{mmol} / \mathrm{l}$ for $2 \mathrm{~h}$ ) brought about in the presence of basal concentrations of insulin and glucagon caused a fall in net hepatic glucose output of $\sim 60 \%$. Suppression of net hepatic glucose output occurred within $15 \mathrm{~min}$ of initiation of hyperglycemia, with maximal suppression being achieved within $30 \mathrm{~min}$ $(6,7)$. Net hepatic glucose output in control dogs, in which euglycemia was maintained, remained essentially unchanged $(6,7)$. Similarly, in the miniature pig, a gradual rise in plasma glucose from 4.0 to $8.5 \mathrm{mmol} / \mathrm{l}$ with insulin and glucagon maintained at basal concentrations resulted in suppression of tracer-determined endogenous glucose production $\left(\right.$ EndoR $_{a}$ ) by 59\% (8). 
Hyperglycemia also inhibited glucose production in overnight-fasted humans. Saccá et al. (9) infused glucose continuously for $2 \mathrm{~h}$ in the presence of fixed pancreatic hormone concentrations. By $20 \mathrm{~min}$, the plasma glucose concentration had risen from 4.2 to $5.5 \mathrm{mmol} / \mathrm{l}$ and EndoR $_{\mathrm{a}}$ had declined $\sim 80 \%$. EndoR remained 80-93\% suppressed throughout the balance of the infusion period (9). Since the hormones were infused peripherally, the hepatic sinusoidal insulin and glucagon concentrations were undoubtedly below basal. Hypoglucagonemia, in particular, probably contributed to the fall in EndoR .

Recently a series of studies was conducted using overnight-fasted dogs maintained on a pancreatic clamp (infusion of somatostatin plus basal intraportal glucagon and insulin) and infused with glucose to create hyperglycemia ranging from $\sim 7$ to $14 \mathrm{mmol} / \mathrm{l}$ $(10,11,47)$. Net hepatic glucose output decreased significantly within 15-30 min of initiating the glucose infusion, in agreement with the findings of Shulman et al. $(6,7)$. These glucose infusions lasted $3 \mathrm{~h}$, while those of Shulman et al. $(6,7)$ lasted only $2 \mathrm{~h}$. With the more prolonged studies, it was evident that the impact of hyperglycemia on net hepatic glucose output did not plateau; instead net hepatic glucose output gradually decreased. By the last $60 \mathrm{~min}$ of glucose infusion, net hepatic glucose output was clearly inversely related to plasma glucose concentration. Net hepatic glucose output fell $40-50 \%$ when plasma glucose increased from basal $(5.8 \mathrm{mmol} / \mathrm{l})$ to $7.5 \mathrm{mmol} / \mathrm{l}$ and ceased altogether at plasma glucose concentrations $\geq 10.5 \mathrm{mmol} / \mathrm{l}$. A low rate of net hepatic glucose uptake was apparent at higher glucose concentrations (Fig. 1).

The ability of the liver to respond to hyperglycemia is apparently impaired in insulin deficiency, but this impairment is at least partially corrected by insulin treatment. Hepatocytes isolated from untreated diabetic rats demonstrated no suppression of glucose production with media glucose concentrations as high as $22 \mathrm{mmol} /$ $\mathrm{l}$; in contrast, hepatocytes from normal and insulintreated diabetic rats exhibited decreases in net glucose production with media glucose of $11 \mathrm{mmol} / \mathrm{l}$ and net uptake of glucose with $16.6 \mathrm{mmol} / \mathrm{l}$ media glucose (12). Similarly, no suppression of net splanchnic glucose output was observed in insulin-deprived insulin-dependent diabetic subjects when their arterial blood glucose concentrations were increased from 14 to $16 \mathrm{mmol} / \mathrm{l}$ by continuous infusion of glucose at $11.1 \mu \mathrm{mol} / \mathrm{kg}$ per min (13). On the other hand, individuals with insulin- and non-insulin-dependent diabetes who underwent euinsulinemic, three-step hyperglycemic clamp studies (glucose concentrations 2.8, 5.6, and $11.2 \mathrm{mmol} / \mathrm{l}$ greater than basal) exhibited decreases in hepatic glucose production which were no different from those in normal subjects matched for body mass index and age (14).

The question of whether the liver itself is responsible for the nonhormonally mediated changes in hepatic

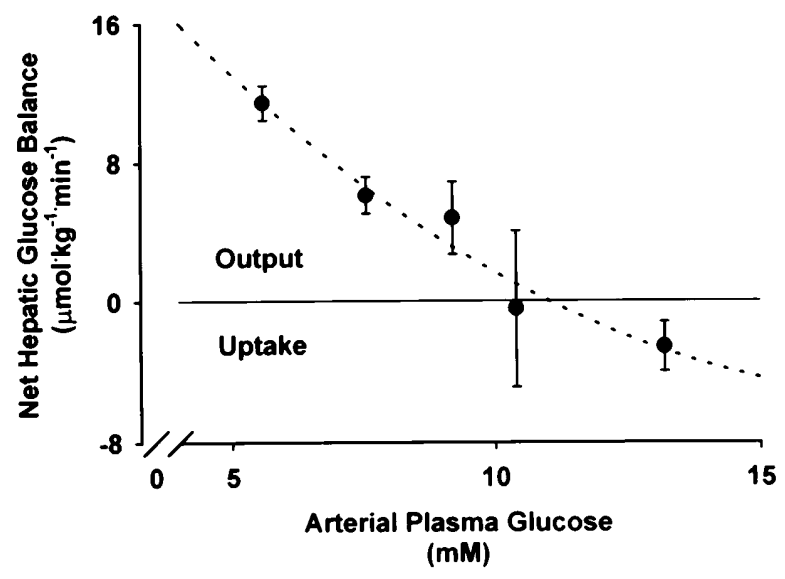

Figure 1 Net hepatic glucose balance in conscious overnight-fasted dogs studied at basal glycemia $(5.5 \mathrm{mmol} / \mathrm{l})$ and increasing levels of hyperglycemia in the presence of somatostatin and basal concentrations of insulin and glucagon. Data are means \pm S.E.M. $n=5$ at each glycemic level. The dotted line represents the curve of best fit, a second order regression $\left(r^{2}=0.96\right)$. Data compiled from references $(10,11,48)$.

glucose output in response to hyperglycemia, or whether the changes are mediated by neural signals or a combination of neural mediation and independent hepatic action, remains unresolved. Electrical stimulation of the lower portion of the cut end of the vagus nerve increased hepatic glycogen synthase activity and deposition of glycogen and decreased net hepatic glucose output in rabbits (15). Niijima (16) demonstrated that intravenous injection of glucose increased the efferent firing rate in the hepatic branch of the vagus nerve in rats, and that the firing rate was directly related to the arterial blood glucose concentration over a range of physiologic concentrations (3.3-25.0 mmol/l). On the other hand, sympathetic stimulation was shown to activate glycogen phosphorylase and glucose-6phosphatase (G-6-Pase) in rabbits (17) and to deplete hepatic glycogen reserves and increase net hepatic glucose output in calves (18). In $18 \mathrm{~h}$ fasted conscious dogs, infusion of somatostatin, 4-fold basal insulin, basal glucagon, and glucose to increase arterial glucose concentrations to $9-11 \mathrm{mmol} / \mathrm{l}$ was associated with a $38 \%$ reduction in hepatic norepinephrine spillover (19). Reduction in sympathetic tone may serve, in combination with increased vagal input, to enhance net hepatic glucose uptake during physiologic hyperglycemia.

\section{Glycogenolysis and gluconeogenesis in the autoregulatory response to hyperglycemia}

Hepatic glucose output is determined by the rates of net glycogenolysis, gluconeogenesis, and glucose cycling between glucose and G-6-Pase. Under usual conditions (i.e. postprandially or after a relatively brief fast), the nonhormonal response to hyperglycemia 
apparently operates primarily through modulation in the rates of glycogenolysis and glucose cycling, rather than gluconeogenesis.

In $6 \mathrm{~h}$ fasted rats infused for $2 \mathrm{~h}$ with somatostatin and studied in the presence of basal insulin, basal glucagon, and $\sim 2$-fold basal plasma glucose, Rossetti et al. (20) observed that hepatic glucose production was decreased 58\%. Glycogenolysis was almost completely inhibited, accounting for $89 \%$ of the decrease in hepatic glucose production. Gluconeogenesis was not significantly changed, and glucose cycling was increased by 2.5-fold when comparison was made with rats studied under euglycemic conditions (20).

Shiota et al. (21) examined rat livers perfused with basal insulin and glucagon, as well as glucose at a concentration of $5.8,12.8$, or $24.4 \mathrm{mmol} / \mathrm{l}$. Net hepatic glucose output was inversely related to the perfusate glucose concentration, both in the presence of low glucagon concentrations $(11 \mathrm{ng} / \mathrm{l})$ and when the perfusate glucagon concentration was increased to $88 \mathrm{ng} / \mathrm{l}$. There was no change in phosphorylase $a$ activity with increasing perfusate glucose concentration; instead, the results were consistent with an increase in glucose cycling (21).

Petersen et al. (22) used ${ }^{13} \mathrm{C}$-nuclear magnetic resonance spectroscopy to assess rates of glycogenolysis in normal overnight-fasted humans during a $6 \mathrm{~h}$ infusion of somatostatin and basal insulin. The plasma glucose concentrations were maintained at either 5 or $10 \mathrm{mmol} / \mathrm{l}$. In comparison to the euglycemic subjects, the hyperglycemic individuals exhibited a $92 \%$ reduction in net hepatic glycogenolysis, primarily because of decreased flux through phosphorylase (22).

Studies in overnight-fasted conscious dogs (which have substantial stores of hepatic glycogen) confirmed the decrease in glycogenolysis in response to hyperglycemia (47). Dogs received somatostatin and basal amounts of intraportal glucagon and insulin while glucose was infused peripherally to increase the arterial glucose level $\sim 2$-fold in a gradual manner. Net hepatic glucose output ceased, and very slight net hepatic glucose uptake was evident (change in net hepatic glucose balance was $13 \mu \mathrm{mol} / \mathrm{kg}$ per min). The change in net hepatic glucose balance could be accounted for by the $78 \%$ reduction in net hepatic glycogenolysis, plus an 11-fold increase in hepatic glucose uptake. As in the studies of Rossetti et al. (20), gluconeogenesis was largely unaffected. Total net hepatic uptake of gluconeogenic precursors (a close but conservative estimate of the true gluconeogenic rate (47)) did not change significantly from baseline.

Normal humans overfed with carbohydrate for 5 days exhibited impairment of hepatic autoregulation (23). In spite of a 2.5-fold increase in plasma insulin concentrations, the 15 overfed subjects demonstrated significant increases (compared with their pre-overfeeding values) in plasma glucose and glucose $\mathrm{R}_{\mathrm{a}}$ after a $10 \mathrm{~h}$ fast. These changes occurred in the overfed individuals despite a $>50 \%$ increase in glucose cycling and a $42 \%$ decrease in the rate of gluconeogenesis $(P<0.01$ vs pre-overfeeding for both changes). Thus these data suggest that a failure to suppress glycogenolysis was responsible for the increase in hepatic glucose output with overfeeding (23).

Even though it appears that changes in the rate of glycogenolysis play a primary role in the autoregulatory response of nondiabetic humans or animals when ample glycogen stores are present, evidence suggests that the liver is also capable of altering its rate of gluconeogenesis in response to changes in glycemia. In dogs studied after a $36 \mathrm{~h}$ fast, when glycogen concentrations were approaching a stable minimum, hyperglycemia was sufficient to induce changes in gluconeogenesis (7). After $2 \mathrm{~h}$ of hyperglycemia $(12 \mathrm{mmol} / \mathrm{l})$ in the presence of basal concentrations of insulin and glucagon, net hepatic fractional extraction of alanine had fallen by $\sim 20 \%$, and the conversion of $\left[{ }^{14} \mathrm{C}\right]$ alanine and $\left[{ }^{14} \mathrm{C}\right] \mathrm{lac}-$ tate into glucose was reduced by $\sim 60 \%$ (7). The decrease in the extraction of gluconeogenic precursors was not sufficient to account for all of the decrease in gluconeogenesis, implying an inhibitory effect of hyperglycemia on the hepatic gluconeogenic process (7).

Hellerstein et al. (24) also observed a decrease in the rate of gluconeogenesis in vivo in response to hyperglycemia. Those authors examined the response of $24 \mathrm{~h}$ fasted rats to intravenous infusion of glucose at 83 or $166 \mu \mathrm{mol} / \mathrm{kg}$ per $\min$ for $4-8 \mathrm{~h}$ (resultant plasma glucose concentrations not reported). Hepatic glucose production declined by $45-53 \%$ in response to glucose infusion, with an increase in glucose cycling accounting for more than half of the reduction in hepatic glucose production at the higher glucose infusion rate. Absolute rates of gluconeogenesis fell $\sim 50 \%$ during both glucose infusions, with glucose recycling accounting for almost all of the gluconeogenic carbon utilized. Apparently hyperglycemia was able to suppress the uptake of gluconeogenic substrates by the liver (24).

In vitro data provide conflicting evidence in regard to the question of whether gluconeogenesis is suppressed in response to hyperglycemia. Using isolated rat hepatocytes, Sánchez-Gutiérrez et al. (25) demonstrated a significant inverse relationship between gluconeogenesis from lactate and pyruvate and the glucose concentration of the medium (with media glucose concentrations ranging from 2.5 to $30 \mathrm{mmol} / \mathrm{l}$ ). Similarly, Ruderman \& Herrera (5) observed a 20\% decrease in gluconeogenesis from alanine in perfused livers from overnight-fasted rats when the perfusate glucose was increased from 8.3 to $16.7 \mathrm{mmol} / \mathrm{l}$. On the other hand, Exton \& Park (2) failed to demonstrate any difference in the rate of gluconeogenesis from lactate in livers from $18-22 \mathrm{~h}$ fasted rats (which would have been virtually free of glycogen) when they were perfused with 12 vs $19 \mathrm{mmol} / \mathrm{l}$ glucose. Similarly, Davidson (12) found that isolated hepatocytes from normal rats incubated with $10 \mathrm{mmol} / \mathrm{l}$ pyruvate exhibited no suppression of gluconeogenesis when the glucose concentration in the 
medium was increased from 5.6 to $22.2 \mathrm{mmol} / \mathrm{l}$; instead, glycogenolysis decreased $75-80 \%$ and glycolysis increased $500 \%$. Differences in study conditions can explain most of the apparent discrepancies in these reports. Exton \& Park (2) used a saturating amount of lactate $(30 \mathrm{mmol} / \mathrm{l})$, which would have made it difficult to detect a small inhibition of gluconeogenesis. Conversely, it is possible that gluconeogenic substrates were limiting in the studies of Sánchez-Gutiérrez et al. (25) and Ruderman \& Herrera (5). In the first instance, the incubation medium provided $2 \mathrm{mmol} / \mathrm{l}$ lactate and $0.2 \mathrm{mmol} / \mathrm{l}$ pyruvate (25), while in the second, the perfusate supplied $1 \mathrm{mmol} / \mathrm{l}$ alanine (5). The duration of fasting (and thus the mass of glycogen available) may also determine whether suppression of gluconeogenesis occurs during hyperglycemia, or whether suppression of glycogenolysis is the primary response. Neither Sánchez-Gutiérrez et al. (25) nor Davidson (12) specified how long the rats had been fasted prior to study. Davidson's data, however, indicate that substantial amounts of glycogen were present in the hepatocytes in the basal state, implying that the rats could have been fasted for no more than a few hours (12).

\section{Mechanisms of autoregulation in response to hyperglycemia}

The key molecular and enzymatic changes associated with autoregulation are only partially elucidated. In vivo data obtained from hyperglycemic rats (20) and humans (22) with basal insulin and glucagon concentrations indicate that a decrease in the activity of hepatic phosphorylase, or a decrease in phosphorylase activity accompanied by an increase in glycogen synthase activity, may explain the decrease in the rate of net glycogenolysis. Rossetti et al. (20) found that the increase in glucose cycling in the hyperglycemic animals was proportional to the increase in the plasma glucose concentration, suggesting that substrate availability was the primary stimulus for the increase in glucose cycling. Alternatively, changes in G-6-Pase could explain the enhancement in glucose cycling in response to hyperglycemia. Incubation of primary hepatocytes with glucose was observed to increase G-6-Pase mRNA 20-fold by $48 \mathrm{~h}$, with maximal stimulation obtained with glucose concentrations as low as $11 \mathrm{mmol} / \mathrm{l}(26)$.

Insulin's importance in the autoregulatory response to hyperglycemia probably results from its effect on the maintenance of glucokinase and glycogen synthase activity within the liver $(12,27)$. The hepatic glucose transporters GLUT-1 and GLUT-2 are membrane bound, and insulin is not required for their translocation (28). GLUT-1 has a low $K_{\mathrm{m}}$ for glucose $(\sim 5 \mathrm{mmol} / \mathrm{l})$, while GLUT-2 has a high $K_{\mathrm{m}}(\sim 15 \mathrm{mmol} / \mathrm{l})$. Because of GLUT-2's high $K_{\mathrm{m}}$ for glucose, the blood glucose concentration is the primary determinant of glucose transport via GLUT-2 (28). Phosphorylation of glucose in the liver is primarily performed by glucokinase, which undergoes transcriptional regulation by a glucoseindependent action of insulin (27). Some genes related to hepatic carbohydrate metabolism (most notably pyruvate kinase) contain glucose response elements and are activated by glucose in an insulin-independent manner, but glucose must be phosphorylated prior to the activation of gene transcription (29). Similarly, the most important effector of glycogen synthase is glucose6-phosphate (30). Thus glucose can stimulate its own intrahepatic metabolism, but even these insulinindependent' processes require insulin for the critical phosphorylation step. Glucokinase and glycogen synthase activities have been observed to be markedly reduced in chronic insulin deficiency $(20,27,31)$. Diminished glucokinase and glycogen synthase activities, coupled with unimpaired phosphorylase $a$ activity, were found to contribute to enhancement of the glycogenolytic rate and maintenance of an elevated rate of basal hepatic glucose production in rats made chronically hyperglycemic and hypoinsulinemic by partial pancreatectomy (20).

In summary, in vivo evidence indicates that under usual circumstances (i.e. when hepatic glycogen stores are present) hyperglycemia inhibits hepatic glucose production primarily through a decrease in the glycogenolytic rate and an increase in glucose cycling $(20-22,47)$. When glycogen reserves are largely depleted, and the individual is relying more heavily upon gluconeogenesis to maintain hepatic glucose output, autoregulation may impact on the rate of gluconeogenesis (7). Evidence appears to support a multifactorial basis for autoregulation. Figure 2 illustrates some of the steps in hepatic glucose metabolism which are likely to be involved in this process.

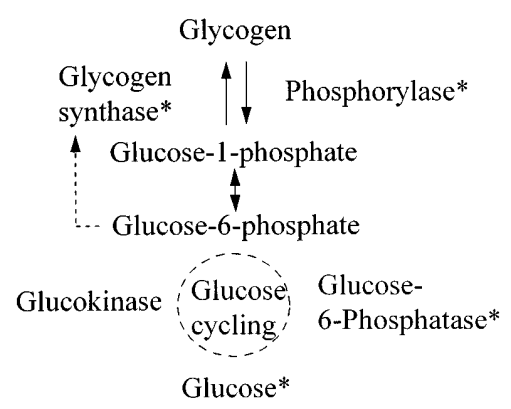

Figure 2 Simplified diagram of relevant steps in hepatic carbohydrate metabolism, with likely sites for autoregulatory actions marked with asterisks. Either an increase in the substrate (glucose) supply or G-6-Pase activity could promote glucose cycling. Glucose-6phosphate is the most potent enhancer of glycogen synthase activity (as shown by the dotted line), and the balance between the activities of glycogen synthase and phosphorylase determine whether glycogen synthesis or glycogenolysis is the dominant process. 


\section{Autoregulation in response to hypoglycemia}

Ideally examination of the autoregulatory phenomenon would occur in the absence of pancreatic hormones or in the presence of only basal hormone concentrations. However, it is virtually impossible to induce hypoglycemia under euinsulinemic conditions without using pharmacologic agents which would alter hepatic glucose production and thus interfere with assessment of the autoregulatory response. Therefore most in vivo investigations of autoregulation during hypoglycemia have been carried out in the presence of insulin-induced hypoglycemia.

In one of the first investigations in vivo, Saccá et al. (32) administered a continuous $3 \mathrm{~h}$ infusion of insulin to conscious overnight-fasted dogs; this resulted in a 3-fold increase in plasma insulin concentrations and a slow fall in plasma glucose, stabilizing at $\sim 2.2 \mathrm{mmol} / \mathrm{l}$. After 10 min of insulin infusion, EndoR $\mathrm{a}_{\mathrm{a}}$ had fallen by $50 \%$, from 20-100 min; however, EndoR $\mathrm{a}_{\mathrm{a}}$ rose steadily. Within 30 min after the insulin infusion began (and at every time thereafter), EndoR $\mathrm{a}_{\mathrm{a}}$ was significantly higher than at $10 \mathrm{~min}$, and it had reached pre-infusion levels by $50 \mathrm{~min}$. The reversal of the insulin inhibition of EndoR $_{\mathrm{a}}$ began 20-50 min prior to measurable changes in counterregulatory hormone secretion (32). Thus the decline in blood glucose appeared to initiate changes in glucoregulation by mechanisms other than increases in concentrations of counterregulatory hormones. There have been major improvements in tracer methodology since the publication of the work of Saccá et al. (32), which call into question the more quantitative aspects of the study. Nevertheless, their findings suggested that autoregulation contributes to the response to hypoglycemia in vivo and this stimulated further investigations.

In another series of experiments in the conscious dog, Frizzell et al. (33) examined three groups of animals receiving an insulin infusion which raised circulating insulin concentrations 20-fold basal. One group was allowed to develop hypoglycemia (to permit the investigators to quantify the counterregulatory response to the resulting glycemic level, $\sim 2.2 \mathrm{mmol} / \mathrm{l}$ ), one group received a glucose infusion to maintain euglycemia (and prevent the rise in counterregulatory hormones), and one group received a glucose infusion to maintain euglycemia plus infusions of glucagon, epinephrine, norepinephrine, and cortisol to mimic the counterregulatory hormone profile observed in the hypoglycemic group. In contrast to the euglycemic group without hormone replacement, the euglycemic group with counterregulatory hormone replacement exhibited an increase in EndoR $\mathrm{a}_{\mathrm{a}}$ during the infusion period, but the increase was only $50 \%$ of that apparent in the hypoglycemic group (33). Thus, the response to hypoglycemia appeared to involve some factor(s) in addition to counterregulatory hormone secretion. The possibilities included a change in autonomic neural activity, the presence of hepatic autoregulation, or a change in hepatic sensitivity to the counterregulatory hormones in the presence of hypoglycemia. This study led to further work to discriminate between these three variables.

Evidence indicates that autoregulation plays a more significant role in the response to severe hypoglycemia than to moderate hypoglycemia $(34,35)$. Hansen et al. (36) studied human volunteers receiving infusions of: (i) somatostatin (to suppress glucagon and growth hormone secretion), insulin ( $\sim 2$-fold basal), and glucose to maintain euglycemia, or (ii) somatostatin, insulin, glucose, and pharmacologic blockers of epinephrine and norepinephrine action. When glucose infusion was decreased to allow the plasma glucose to fall to $3.6 \mathrm{mmol} / \mathrm{l}$ (a moderate hypoglycemic stimulus), glucose production increased $7.2 \mu \mathrm{mol} / \mathrm{kg}$ per min in the group not receiving adrenergic blockade but did not change in the blockade group; when glucose was then infused to bring plasma glucose back to basal, glucose production decreased $8.3 \mu \mathrm{mol} / \mathrm{kg}$ per min (thus reversing all of the increase observed in response to hypoglycemia) and $2.8 \mu \mathrm{mol} / \mathrm{kg}$ per min in the nonblockade and the blockade groups respectively. The investigators concluded that counterregulatory hormones were responsible for almost all of the increase in glucose production observed, with autoregulation accounting for a maximum of $0-30 \%$ of the change in glucose production (36).

Bolli et al. (34) used pharmacologic agents to inhibit the secretion of glucagon, growth hormone, and cortisol and to block the actions of epinephrine and norepinephrine during insulin-induced hypoglycemia. They replaced glucagon and growth hormone at basal levels, and infused insulin to induce moderate $(2.8 \mathrm{mmol} / \mathrm{l})$ or more severe $(1.7 \mathrm{mmol} / \mathrm{l})$ hypoglycemia in human subjects. In the group with moderate hypoglycemia, EndoR $\mathrm{a}_{\mathrm{a}}$ was rapidly suppressed $\sim 70 \%$ and did not rise throughout the period of hyperinsulinemia. Glucose production was similarly suppressed when euglycemia was maintained by glucose infusion during the period of hyperinsulinemia (again in the presence of the pharmacologic blockers). In contrast, after an initial suppression of glucose production in the severely hypoglycemic group, Endo $\mathrm{R}_{\mathrm{a}}$ rose so that it was 2-fold greater than during euglycemia or moderate hypoglycemia. The investigators concluded that autoregulation was a component of the response to severe hypoglycemia (and was responsible for $\sim 2.8 \mu \mathrm{mol} / \mathrm{kg}$ per min of glucose production) but had little importance at plasma glucose levels $\geq 2.8 \mathrm{mmol} / \mathrm{l}$ (34).

The studies of Hansen et al. (36) and Bolli et al. (34) in human subjects were limited by the investigators' inability to control counterregulatory hormones completely. In the studies of Hansen et al. (36), for example, cortisol and growth hormone concentrations increased $166-221 \mathrm{nmol} / \mathrm{l}$ and $1 \mu \mathrm{g} / \mathrm{l}$ respectively during hypoglycemia. Moreover, adrenergic blockade does not have the same metabolic effect as epinephrine deficiency 
resulting from adrenalectomy (37). Connolly et al. (38) therefore conducted studies to define more precisely the relationship between plasma glucose concentrations and glucose metabolism in the conscious dog. Adrenalectomized dogs (lacking cortisol and epinephrine) were infused with somatostatin, as well as a high dose of intraportal insulin, resulting in arterial insulin concentrations of $\sim 2000 \mathrm{pmol} / \mathrm{l}$. Glucose was infused peripherally as needed to achieve four different glycemic plateaus in each $\operatorname{dog}(5.9,3.4,2.5$, and $1.7 \mathrm{mmol} / \mathrm{l})$. There were no detectable circulating concentrations of glucagon, epinephrine, or cortisol. (Growth hormone could not be measured due to the lack of a sensitive assay for canine growth hormone.) The liver exhibited net uptake of glucose $(7.5 \mu \mathrm{mol} / \mathrm{kg}$ per min) during the euglycemic, hyperinsulinemic period $(5.9 \mathrm{mmol} / \mathrm{l}$ glucose) and then changed to a net output of $3.0 \mu \mathrm{mol} / \mathrm{kg}$ per min at the first hypoglycemic level $(3.4 \mathrm{mmol} / \mathrm{l})$. Net hepatic output of glucose increased progressively with increasing severity of hypoglycemia, reaching $6.9 \mu \mathrm{mol} / \mathrm{kg}$ per min during the hypoglycemic nadir (Fig. 3). Tracer-determined EndoR $\mathrm{a}_{\mathrm{a}}$ corroborated the changes in net hepatic glucose output. Plasma norepinephrine concentrations increased with each successive decrease in plasma glucose and were $\sim 2$-fold higher at a glucose concentration of $1.7 \mathrm{mmol} / \mathrm{l}$ than at $5.9 \mathrm{mmol} / \mathrm{l}$. The effect of this change in norepinephrine on glucose metabolism would have been minor, if the change in norepinephrine had been restricted to circulating concentrations only. A 30-fold basal increase in hepatic sinusoidal norepinephrine was required to increase net hepatic glucose output as much as Connolly et al. (38) observed at the $1.7 \mathrm{mmol} / \mathrm{l}$ hypoglycemic plateau (39). Unfortunately, the rise in circulating norepinephrine

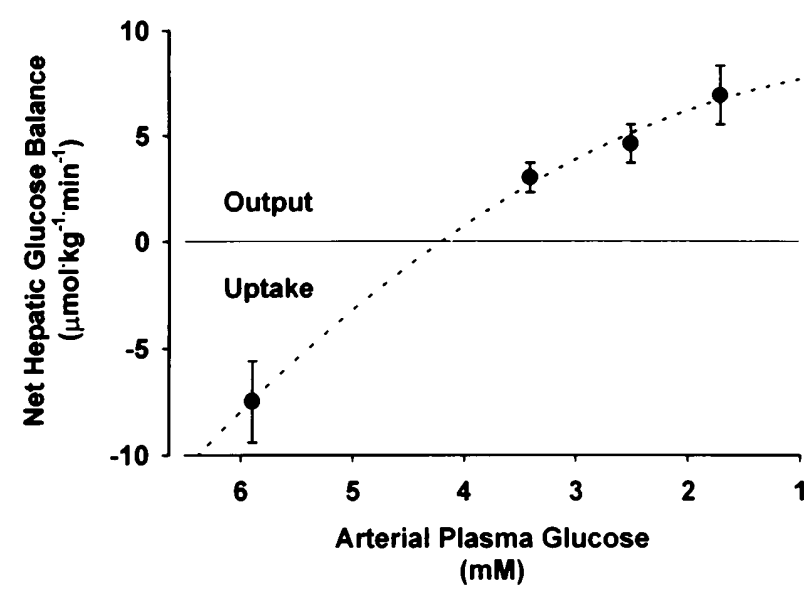

Figure 3 Net hepatic glucose balance in conscious overnight-fasted adrenalectomized dogs studied at basal glycemia $(5.9 \mathrm{mmol} / \mathrm{l})$ and three different severities of hypoglycemia in the presence of somatostatin and hyperinsulinemia $(\sim 2000 \mathrm{pmol} / \mathrm{l})$. Data are means \pm S.E.M. $n=7$. The dotted line represents the curve of best fit, a second order regression $\left(r^{2}=0.99\right)$. Data derived from reference (38). observed by Connolly et al. (38) reflected spillover from the synaptic clefts and thus an enhancement of sympathetic signaling, rather than a hormonal change. There are no data to indicate what effect a neural signal of this magnitude (i.e. one which would create a 2 -fold rise in circulating norepinephrine) would have on net hepatic glucose output. Nevertheless, the work of Connolly et al. (38) conclusively demonstrated in vivo that nonhormonal mechanisms (neural and/or autoregulatory) stimulate net hepatic glucose output in response to insulin-induced hypoglycemia. In addition, comparison of the data of Connolly et al. (38) with those of Frizzell et al. (33) made it possible to quantify the impact of nonhormonal mechanisms on the response to a glycemic level of $\sim 2.5 \mathrm{mmol} / \mathrm{l}$ (a glucose concentration common to both investigations). Nonhormonal mechanisms, possibly including autoregulation, could account for approximately one-quarter of the change in net hepatic glucose balance observed at $2.5 \mathrm{mmol} / \mathrm{l}$ glucose (38).

The brain bears the responsibility for directing most of the rise in counterregulatory hormones seen in response to hypoglycemia (40), but it also may affect hepatic glucose metabolism directly via neural connections. It has been demonstrated in several species that electrical stimulation of the splanchnic or hepatic nerves results in hyperglycemia, in spite of adrenalectomy and pancreatectomy $(41,42)$. Stimulation of the ventromedial hypothalamus results in enhancement of hepatic glycogenolysis and gluconeogenesis $(43,44)$. To separate the possible effects of glucopenia at the level of the central nervous system from direct effects of glucopenia on the liver Connolly et al. (45) conducted further studies in the conscious dog model. The dogs underwent adrenalectomy 2 weeks prior to experimentation, as in the previous investigation (38). During the studies, dogs received somatostatin, along with intraportal insulin infusion to produce arterial concentrations of $\sim 1500 \mathrm{pmol} / \mathrm{l}$. Glucose was infused peripherally as needed to maintain the plasma glucose level at $\sim 2 \mathrm{mmol} / \mathrm{l}$. One group of dogs also received glucose infusions via both common carotid and vertebral arteries, to maintain euglycemia within the brain, while the second group was allowed to develop brain hypoglycemia. Both groups switched from net hepatic uptake of glucose during the control period (systemic euglycemic hyperinsulinemia) to net hepatic release of glucose during the hypoglycemic period, with no difference in response between groups (change in net hepatic glucose balance $\sim 12 \mu \mathrm{mol} / \mathrm{kg}$ per min in both groups). Thus maintenance of cerebral euglycemia did not prevent the rise in hepatic glucose release caused by the low blood glucose, and consequently liver hypoglycemia must have stimulated net hepatic glucose release (45). The results of this study do not rule out a role for the nervous system in the response observed (i.e. communication by the liver to the brain concerning the hypoglycemia at the level of the liver, with a 
subsequent response by the brain), but they do indicate that an increase in hepatic glucose production can occur in response to hypoglycemia sensed within the liver and in the absence of a change in counterregulatory hormone secretion. The results are all the more impressive in view of the fact that insulin concentrations at the liver were high and should have represented a maximal inhibitory signal to glucose production, yet the liver clearly responded to hypoglycemia.

In a further effort to separate the direct response of the liver from the neurally mediated response to hypoglycemia, the same group of investigators (C C Connolly \& A D Cherrington, unpublished observations) conducted additional studies in six adrenalectomized dogs which had also undergone surgical denervation of the liver. These animals were studied in the same manner as the adrenalectomized dogs described above: somatostatin was infused, hyperinsulinemia was created, cerebral euglycemia was maintained, and glucose was infused peripherally as needed to maintain circulating glucose at $\sim 2 \mathrm{mmol} / \mathrm{l}$. In this case, the change in net hepatic glucose balance during the hypoglycemic period was $6 \mu \mathrm{mol} / \mathrm{kg}$ per min, about half of the change observed in the animals with intact hepatic nerves. Although these data cannot be interpreted in a strictly quantitative sense, it is clear that a portion of the liver's nonhormonally mediated response to hypoglycemia cannot be attributed to direct neural control.

Taken together, the studies described above indicate that nonhormonal mechanisms may be responsible for $25-30 \%$ of the change in hepatic glucose production in response to severe hypoglycemia $(\leq 2.8 \mathrm{mmol} / \mathrm{l})$. This implies that the nonhormonal contribution to glucose production during hypoglycemia is sufficient to meet approximately half of the brain's obligatory glucose requirement (34). Thus, even though nonhormonal mechanisms are responsible for a limited portion of the total hepatic response to hypoglycemia under usual conditions, their contribution is nevertheless physiologically significant since it occurs when there is a critical need for glucose.

\section{Mechanisms involved in hypoglycemic autoregulation}

Autoregulation in response to hypoglycemia apparently can involve both gluconeogenesis and glycogenolysis. Ruderman \& Herrera (5) observed a 35\% increase $(P<0.005)$ in gluconeogenesis from $\left[{ }^{14} \mathrm{C}\right]$ alanine in rat livers perfused with a medium devoid of glucose, as opposed to those perfused with $16.6 \mathrm{mmol} / \mathrm{l}$ glucose. Net glycogenolysis occurred in the livers perfused without glucose, while livers perfused with 8.3 or $16.6 \mathrm{mmol} / \mathrm{l}$ glucose exhibited net glycogen synthesis (both $P<0.001$ vs livers perfused without glucose) (5). When glycogen stores are present, an increase in the rate of glycogenolysis may be the preferred autoregulatory response to hypoglycemia. Livers from fed rats responded to a perfusion without glucose by releasing glucose and lactate into the perfusate, and the loss of tissue glycogen could account for all of the carbon released as glucose and lactate (3).

Enhancement of the rate of gluconeogenesis becomes an important facet of the autoregulatory response when glycogen reserves are low. McCraw et al. (4) examined the response of isolated rat livers to perfusate glucose concentrations ranging from 0 to $16.6 \mathrm{mmol} / \mathrm{l}$ in the presence of $20 \mathrm{mmol} / \mathrm{l}$ lactate. When livers of normal rats fasted for $24 \mathrm{~h}$ (and thus depleted in glycogen) were perfused with medium containing 2.8 and $0 \mathrm{mmol} / \mathrm{l}$ glucose, they exhibited $50 \%$ and $300 \%$ increases respectively in the rate of net glucose production in comparison to the rate observed during perfusion with $5.5 \mathrm{mmol} / \mathrm{l}$ glucose. Perfused livers from $12 \mathrm{~h}$ fasted adrenalectomized rats (which have a block of gluconeogenesis from lactate) demonstrated net uptake of glucose at 5.5 and $2.8 \mathrm{mmol} / \mathrm{l}$ perfusate glucose concentrations, and released only $41 \%$ as much glucose as normal livers when the perfusate was devoid of glucose (4).

\section{Conclusion}

The phenomenon of autoregulation plays an important role in regulation of hepatic glucose metabolism. In the presence of physiologic hyperglycemia (<12 mmol/l) with basal insulin and glucagon concentrations, hepatic glucose production may fall by as much as 60-90\%. Following a relatively brief fast (i.e. overnight in the dog and human), reduction in hepatic glycogenolysis and enhancement of glucose cycling are the primary mechanism by which the autoregulatory response occurs. After a more prolonged fast, when hepatic glycogen concentrations have been reduced to a stable minimum, reduction in the gluconeogenic rate becomes the predominant mechanism of the autoregulatory response. Although the rate of hepatic glucose production is greater in individuals with non-insulin-dependent diabetes (NIDDM) than in persons without NIDDM, it appears that hepatic autoregulation also occurs in NIDDM (46).

The autoregulatory response to insulin-induced hypoglycemia makes its most significant contribution in the presence of very low circulating glucose levels $(\leq 2.8 \mathrm{mmol} / \mathrm{l})$. Autoregulation is capable of significant stimulation of hepatic glucose production in the presence of severe hypoglycemia, providing as much as $3 \mu \mathrm{mol} / \mathrm{kg}$ per min of glucose, with both glycogenolysis and gluconeogenesis potentially contributing to the autoregulatory response. Although in vivo investigations have utilized insulin-induced hypoglycemia to examine the role of hepatic autoregulation, the in vitro data suggest that an autoregulatory response to hypoglycemia does not require insulin. The endocrine system is a dominant force in the response of the liver to hyperglycemia and hypoglycemia, but autoregulation 
provides an important adjunctive mechanism which may become important in the event of severe derangement of circulating glucose levels or impairment of neuroendocrine function.

\section{References}

1 Soskin S, Essex HE, Herrick JF \& Mann FC. The mechanism of the regulation of blood sugar by the liver. American Journal of Physiology 1938124 558-567.

2 Exton JH \& Park CR. Control of gluconeogenesis in liver. I. General features of gluconeogenesis in the perfused liver of rats. Journal of Biological Chemistry 1967242 2622-2636.

3 Glinsmann WH, Hern EP \& Lynch A. Intrinsic regulation of glucose output by rat liver. American Journal of Physiology 1969 216 698-703.

4 McCraw EF, Peterson MJ \& Ashmore J. Autoregulation of glucose metabolism in the isolated perfused rat liver. Proceedings of the Society for Experimental Biology and Medicine $1967126232-236$.

5 Ruderman NB \& Herrera MG. Glucose regulation of hepatic gluconeogenesis. American Journal of Physiology $19682141346-$ 1351.

6 Shulman GI, Liljenquist JE, Williams PE, Lacy WW \& Cherrington AD. Glucose disposal during insulinopenia in somatostatintreated dogs: the roles of glucose and glucagon. Journal of Clinical Investigation 197862 487-491.

7 Shulman GI, Lacy WW, Liljenquist JE, Keller U, Williams PE \& Cherrington AD. Effect of glucose, independent of changes in insulin and glucagon secretion, on alanine metabolism in the conscious dog. Journal of Clinical Investigation 198065 496-505.

8 Müller MI, Möring J \& Seitz HJ. Regulation of hepatic glucose output by glucose in vivo. Metabolism 198837 55-60.

9 Saccá L, Hendler R \& Sherwin RS. Hyperglycemia inhibits glucose production in man independent of changes in glucoregulatory hormones. Journal of Clinical Endocrinology and Metabolism 1978 47 1160-1163.

10 Chu CA, Sindelar DK, Neal DW, Allen EJ, Donahue EP \& Cherrington AD. Comparison of the direct and indirect effects of epinephrine on hepatic glucose production. Journal of Clinical Investigation 199799 1044-1056.

11 Chu CA, Sindelar DK, Neal DW, Allen EJ, Donahue EP \& Cherrington AD. The effects of a selective rise in hepatic sinusoidal norepinephrine on hepatic glucose production are solely attributable to an increase in glycogenolysis. American Journal of Physiology 199837 E162-E171.

12 Davidson MB. Autoregulation by glucose of hepatic glucose balance: permissive effect of insulin. Metabolism 198130 279-284.

13 Wahren J, Felig P, Cerasi E \& Luft R. Splanchnic and peripheral glucose and amino acid metabolism in diabetes mellitus. Journal of Clinical Investigation 197251 1870-1878.

14 Del Prato S, Matsuda M, Simonson DC, Groop LC, Sheehan P, Leonetti F, et al. Studies on the mass action effect of glucose in NIDDM and IDDM: evidence for glucose resistance. Diabetologia $199740687-697$.

15 Shimazu T \& Fujimoto T. Regulation of glycogen metabolism in liver by the autonomic nervous system. IV. Neural control of glycogen biosynthesis. Biochimica et Biophysica Acta 1971252 $18-27$.

16 Niijima A. Blood glucose levels modulate efferent activity in the vagal supply to the rat liver. Journal of Physiology 1985364105 112.

17 Shimazu T \& Amakawa A. Regulation of glycogen metabolism in liver by the autonomic nervous system. II. Neural control of glycogenolytic enzymes. Biochimica et Biophysica Acta 1968165 335-348.

18 Edwards $\mathrm{AV}$ \& Silver M. The glycogenolytic response to stimulation of the splanchnic nerves in adrenalectomized calves. Journal of Physiology 1970211 109-124.
19 Ikeda T, Scott M, Emshwiller MG, Monohan MT, Allen E, Sindelar DK et al. Hyperglycemia reduces sympathetic nervous signaling to the liver and gut in the dog. Diabetes 199746 (Suppl 1) 232A.

20 Rossetti L, Giaccari A, Barzilai N, Howard K, Sebel G \& Hu M. Mechanism by which hyperglycemia inhibits hepatic glucose production in conscious rats: implications for the pathophysiology of fasting hyperglycemia in diabetes. Journal of Clinical Investigation $1993921126-1134$.

21 Shiota M, Green R, Colburn CA, Mitchell G \& Cherrington AD. Inability of hyperglycemia to counter the ability of glucagon to increase net glucose output and activate glycogen phosphorylase in the perfused rat liver. Metabolism 199645 481-485.

22 Petersen KF, Laurent D, Rothman DL, Cline GW \& Shulman GI. Mechanism by which insulin inhibits net hepatic glycogenolysis in man. Diabetologia 199740 (Suppl 1) A25.

23 Clore JN, Helm ST \& Blackard WH. Loss of hepatic autoregulation after carbohydrate overfeeding in normal man. Journal of Clinical Investigation 199596 1967-1972.

24 Hellerstein MK, Neese RA, Schwarz JM, Turner S, Faix D \& Wu K. Altered fluxes responsible for reduced hepatic glucose production and gluconeogenesis by exogenous glucose in rats. American Journal of Physiology 1997272 E163-E172.

25 Sánchez-Gutiérrez JC, Lechuga CG, Sánchez-Arias JA, Samper B \& Felíu JE. Impairment of the modulation by glucose of hepatic gluconeogenesis in the genetically obese (fa/fa) Zucker rat. Endocrinology 1995136 1877-1884.

26 Argaud D, Kirby TL, Newgard CB \& Lange AJ. Stimulation of glucose-6-phosphatase gene expression by glucose and fructose2,6-bisphosphate. Journal of Biological Chemistry 1997272 12854-12861.

27 Vaulont S \& Kahn A. Transcriptional control of metabolic regulation genes by carbohydrates. FASEB Journal 19948 28-35.

28 Pessin JE \& Bell GI. Mammalian facilitative glucose transporter family: structure and molecular regulation. Annual Review of Physiology 199254 911-930.

29 Lefrançois-Martinez AM, Diaz-Guerra MJM, Vallet V, Kahn A \& Antoine B. Glucose-dependent regulation of the L-pyruvate kinase gene in a hepatoma cell line is independent of insulin and cyclic AMP. FASEB Journal 19948 89-96.

30 Villar-Palasi C \& Guinovart JJ. The role of glucose 6-phosphate in the control of glycogen synthase. FASEB Journal $199711544-$ 558 .

31 Brichard SM, Henquin JC \& Girard J. Phlorizin treatment of diabetic rats partially reverses the abnormal expression of genes involved in hepatic glucose metabolism. Diabetologia 199336 292-298.

32 Saccá L, Cryer PE \& Sherwin RS. Blood glucose regulates the effects of insulin and counterregulatory hormones on glucose production in vivo. Diabetes 197928 533-536.

33 Frizzell RT, Hendrick GL, Brown LL, Lacy DB, Donahue EP, Carr RK et al. Stimulation of glucose production through hormone secretion and other mechanisms during insulin-induced hypoglycemia. Diabetes 198837 1531-1541.

34 Bolli G, De Feo P, Perriello G, De Cosmo S, Ventura M, Campbell P et al. Role of hepatic autoregulation in defense against hypoglycemia in humans. Journal of Clinical Investigation 1985 75 1623-1631.

35 Cryer PE. Glucose counterregulation: prevention and correction of hypoglycemia in humans. American Journal of Physiology 1993 264 E149-E155.

36 Hansen I, Firth R, Haymond M, Cryer P \& Rizza R. The role of autoregulation of the hepatic glucose production in man: response to a physiologic decrement in plasma glucose. Diabetes $198635186-191$.

37 Tse T, Clutter W, Shah S \& Cryer P. Mechanisms of postprandial glucose counterregulation in man: physiologic roles of glucagon and epinephrine vis- $a$-vis insulin in the prevention of hypoglycemia later after glucose ingestion. Journal of Clinical Investigation 198372 278-286.

38 Connolly CC, Adkins-Marshall BA, Neal DW, Pugh W, Jaspan JB \& 
Cherrington AD. Relationship between decrements in glucose level and metabolic response to hypoglycemia in absence of counterregulatory hormones in the conscious dog. Diabetes 1992 41 1308-1319.

39 Connolly CC, Steiner KE, Stevenson RW, Neal DW, Williams PE, Alberti KGMM et al. Regulation of glucose metabolism by norepinephrine in the conscious dog. American Journal of Physiology 1991261 E764-E772.

40 Biggers DW, Myers SR, Neal D, Stinson R, Cooper NB, Jaspan JB et al. Role of brain in counterregulation of insulin-induced hypoglycemia in dogs. Diabetes 198937 7-16.

41 Edwards AV. The hyperglycaemic response to stimulation of the hepatic sympathetic innervation in adrenalectomised cats and dogs. Journal of Physiology 1972220 697-710.

42 Edwards AV \& Silver M. Comparison of the hyperglycaemic and glycogenolytic responses to catecholamines with those to stimulation of the hepatic sympathetic innervation in the dog. Journal of Physiology 1972223 571-593.

43 Shimazu T, Fukuda A \& Ban T. Reciprocal influences of the ventromedial and lateral hypothalamic nuclei on blood glucose level and liver glycogen content. Nature $19662101178-1179$.

44 Shimazu T \& Matsushita H. Role of the norepinephrine- and acetylcholine-sensitive neurons of the hypothalamus in the regulation of hepatic metabolism. In Catecholamines: Basic and Clinical Frontiers, vol 1, pp 980-982. Eds E Usdin, IJ Kopin \& J Barchas. New York: Pergamon Press, 1978.

45 Connolly CC, Myers SR, Neal DW, Hastings JR \& Cherrington AD. In the absence of counterregulatory hormones, the increase in hepatic glucose production during insulin-induced hypoglycemia in the dog is initiated in the liver rather than the brain. Diabetes $1996451805-1813$.

46 Tappy L. Regulation of hepatic glucose production in healthy subjects and patients with non-insulin-dependent diabetes mellitus. Diabete et Metabolisme 199521 233-240.

47 Sindelar DK, Chu CA, Venson P, Donahue EP, Neal DW \& Cherrington AD. Basal hepatic glucose production is regulated by the portal vein insulin concentration. Diabetes 1998 (In Press).

48 Sindelar DK, Chu CA, Neal DW \& Cherrington AD. The interaction between equal increments in peripheral and portal vein insulin in regulating hepatic glucose production in the conscious dog. American Journal of Physiology 273 E972-E980.

Received 13 October 1997

Accepted 2 December 1997 